\title{
FRECUENCIA DEL CUARTO CONDUCTO YVARIACIONES ANATÓMICAS EN SEGUNDAS Y TERCERAS MOLARES SUPERIORES MEDIANTE TOMOGRAFÍA COMPUTARIZADA DE
} HAZ CÓNICO

FREQUENCY OF MESIOBUCAL CANAL AND ANATOMICAL VARIATIONS IN THE MAXILLARY SECOND AND THIRD MOLAR USING CONE BEAM COMPUTED TOMOGRAPHY

\section{RESUMEN}

Calero-Hinostroza, Gisela ${ }^{1}$ Gonzales-Gonzales, Ronald ${ }^{2}$ García-Rupaya, Carmen Rosa ${ }^{3}$

Objetivo: Evaluar la frecuencia del cuarto conducto y variaciones anatómicas en segundas y terceras molares superiores mediante tomografía de haz cónico volumétrico.

Materiales y método: Se incluyeron segundos o terceros molares superiores de pacientes en 120 tomografías de haz cónico volumétrico, recolectadas entre enero y diciembre de 2016. Las tomografías se obtuvieron con un tomógrafo Point 3D Combi $500 \mathrm{~S}$ con sensor de FOV de $12 \times 9 \mathrm{~cm}$. Se realizó el análisis mediante SPSS y se realizó la prueba de Chi-cuadrado de Pearson.

Resultados: La frecuencia del cuarto conducto de las segundas molares superiores fue de $40 \%$ y en terceras molares superiores fue de 4,8\%. La variante tipo 8 fue de $47 \%$ y la variante 9 fue de $38,8 \%$ en segundas molares superiores. La variante 3 fue de $34,9 \%$ y la variante tipo 1 fue de $28,1 \%$ en terceras molares superiores. La mayor frecuencia fue de 3 raíces, $86,5 \%$ en segundas molares superiores y en las terceras molares superiores $69,2 \%$ tuvo una sola raíz. La bilateralidad en segundas molares superiores fue de $69,4 \%$ y en terceras molares superiores fue de $69,9 \%$.

Conclusiones: En segundas molares superiores la presencia del cuarto conducto fue de $40 \%$ y en las terceras molares superiores fue $4,8 \%$.

Palabras clave: cuarto conducto, tomografía de haz cónico volumétrico, segunda molar superior, tercera molar superior

\section{ABSTRACT}

Objective: To evaluate the frequency of mesiobucal canal and anatomical variations in the maxillary second molar and maxillary third molar using cone beam computed tomographic (CBCT).

Material and Methods: Maxillary second molars or maxillary third molars of patients were included using cone beam computed tomographic (CBCT) imaging between January and December 2016. CBCT scans were obtained with a Point 3D Combi 500 S tomograph with $12 \times 9 \mathrm{~cm}$ FOV. The SPSS analysis and the Pearson Chi-square test was performed.

Results: The frequency of the MB2 of the maxillary second molar was $40 \%$ and in maxillary third molar was 4,8\%. Type 8 variant was $47 \%$ and variant 9 was 38,8\% in maxillary second molar. The variant 3 was $34,9 \%$ and the type 1 variant was $28,1 \%$ in the maxillary third molar. The highest frequency was 3 roots, $86,5 \%$ in maxillary second molar and in the maxillary third molar $69,2 \%$ a single root. The bilaterality in the maxillary second molar was $69,4 \%$ and in the maxillary third molar was $69,9 \%$.

Conclusions: In the maxillary second molar the presence MB2 was $40 \%$ and in the maxillary third molar was $4.8 \%$.

Keywords: mesiobucal canal, MB2, cone beam, maxillary second molar, maxillary third molar 


\section{INTRODUCCIÓN}

El éxito del tratamiento endodóntico implica un correcto diagnóstico, un buen entendimiento de los principios biológicos, una adecuada preparación y una obturación tridimensional del sistema de conductos radiculares. Para lograr estos objetivos es fundamental que el profesional tenga un profundo conocimiento sobre la morfología interna de dicho sistema.

Una de las mayores causas de fracasos en el tratamiento de conducto es no localizar y tratar todo el sistema de conductos, como resultado de la falta de conocimiento de la anatomía dental externa e interna(1) y a la alta complejidad y variaciones del sistema de conductos, con conductos accesorios, secundarios recurrentes y deltas apicales(2).

Numerosos estudios han demostrado las variaciones morfológicas de las molares superiores, enfatizando la presencia del cuarto conducto. Estas variaciones pueden ser atribuidas a los diferentes métodos usados. Algunos métodos usados son la tinción y diafanización,(3-6) cortes transversales con uso de estereomicroscopio,(7) mediante el uso de fotografía a los cortes transversales,(8) uso de radiografías periapicales en comparación de haz cónico volumétrico(9).

La tomografía de haz cónico volumétrico ha sido introducida para superar las limitaciones de las radiografías convencionales como la distorsión causada por la angulación y superposición de estructuras anatómicas $(10,11)$ así como la detección de reparación apical(12).

Según Kalender et al.(13) evaluaron mediante tomografía de haz cónico volumétrico 373 primeros molares maxilares, de los cuales $97,8 \%$ presentaron 3 raíces; 3,1\% una sola raíz; 1,6\% 4 raíces; 0,53\% 2 raíces. Entre los 438 segundos molares, $89,4 \%$ tenían 3 raíces; 5,9\% tenían 2 raíces; 3,1\% tenían una sola raíz, y 1,3\% tenían 4 raíces. En el estudio de Abarca et al. ${ }^{14}$ estudiaron 1374 dientes mediante tomografía de haz cónico volumétrico, en los primeros molares maxilares (802) y segundos molares superiores (572), la frecuencia del cuarto conducto. En las primeras molares maxilares fue $73,44 \%$ y $42,48 \%$ en segundas molares superiores. La morfología más frecuente en primeros molares maxilares y segundos molares superiores eran Vertucci tipo II y l, respectivamente.

Nikoloudaki et al(15) evaluaron mediante tomografía de haz cónico volumétrico, (410 primeras molares superiores y 402 segundas molares superiores). La gran mayoría de ambas, primeras y segundas molares tenían tres raíces $(89,26 \%$ y $85,07 \%$, respectivamente). La mayor parte de las primeras molares superiores tuvieron cuatro conductos, mientras que la mayoría de las segundas molares tenían tres. También se encontraron otras variaciones morfológicas raras, tales como la fusión de un segundo molar superior con un diente supernumerario.

Silva et al.(16) analizaron y caracterizaron la morfología del conducto radicular de los molares superiores de la población brasileña mediante tomografía de haz cónico volumétrico. Primeros y segundos molares mostraron una mayor prevalencia de 3 raíces separadas, mesiovestibular, distovestibulary palatina, con 1 conducto en cada raíz $(52,87 \%$ y $45,09 \%$, respectivamente). Dos conductos de las raíces mesiovestibulares representados $42,63 \%$ de las primeras molares superiores, mientras que las raíces mesiovestibulares del segundo molar presentan 2 conductos en 34,32\%. 
Altunsoy et al.(17) determinaron la raíz y la morfología del conducto de los primeros molares y segundos maxilares en una subpoblación de Turquía con el uso de tomografía de haz cónico computarizada (CBCT). Tanto en las primeras y segundas molares superiores, la presencia de tres raíces fue la ocurrencia más común. La prevalencia de conductos adicionales (tipo II, III, o mayor) en las raíces mesiovestibulares del primer y segundo molar maxilar fue de aproximadamente $62 \%$ y $37,5 \%$, respectivamente. El tipo I fue el más prevalente en el distovestibular $(99,6 \%)$ y palatino $(99,8 \%)$ de primeros molares maxilares, y en el distovestibular $(99,7 \%)$ y palatina $(99,2 \%)$ raíces de segundos molares superiores.

Hasta donde sabemos, no hay informes sobre frecuencia del cuarto conducto y variaciones anatómicas en la segunda y tercera molar superior mediante la tomografía de haz cónico volumétrico. La pérdida del primer y segundo molar superior hace que el tercer molar deba considerarse un soporte estratégico. Otra indicación para la terapia endodóntica es la presencia de un tercer molar inferior funcional en una arcada con espacio suficiente para la erupción completa y la higiene oral. Desde el punto de vista clínico, los resultados de esta investigación aportarán información necesaria sobre las variaciones anatómicas y así mejorará la práctica clínica disminuyendo los fracasos por desconocimiento de las variantes anatómicas particulares que presentan, contribuyendo de esta manera la realización de un mejor tratamiento endodóntico y con mejor pronóstico.

El conocimiento de la anatomía interna de los conductos radiculares es uno de los determinantes para el éxito de un tratamiento endodóntico. El presente estudio investigará las variantes anatómicas y presencia del cuarto conducto en segundas y terceras molares superiores.

\section{MATERIALES Y MÉTODOS}

Este estudio observacional, descriptivo, retrospectivo y transversal, fue realizado en el centro de Diagnóstico por Imágenes HANNY $X$ en el período de enero y diciembre de 2016, en pacientes entre 18 y 80 años de edad. Para garantizar la confidencialidad de los pacientes no se usaron los nombres, las tomografías fueron codificadas. Fue presentado para su revisión y aprobación a la Comisión de Revisión de Aspectos Metodológicos y Éticos de la carrera de Estomatología de la Universidad Científica del Sur.

Se utilizó la fórmula para estimar una proporción, a un nivel de confianza de $95 \%$, con un margen de error de $5 \%$, dando como resultado 120 tomografías que presenten segundas y terceras molares superiores siendo los criterios de exclusión: ápice abierto, signos de reabsorción apical, cirugía apical, restauraciones metálicas, prótesis fijas, postes, conductos calcificados, evidencia de radicectomías, periodontitis apical, reabsorción externa o interna.

Se evaluó la morfología interna, presencia del cuarto conducto, número de raíces, número de conductos, género, edad y bilateralidad. Las tomografías de haz cónico volumétrico se obtuvieron de un tomógrafo Point 3D Combi $500 \mathrm{~S}$ que usa sensor de FOV (field of view) de $12 \times 9 \mathrm{~cm}$. También posee el sistema de reducción de artefactos metálicos que elimina los "destellos" creados por elementos metálicos permitiendo una imagen diagnóstica más precisa. Cada toma tomográfica fue tomada siguiendo las instrucciones del fabricante.

Luego de la autorización, la especialista en radiología oral y maxilofacial capacitó y calibró en el manejo del Real Scan, que es el programa de visualización de tomografías a la investigadora del estudio. 


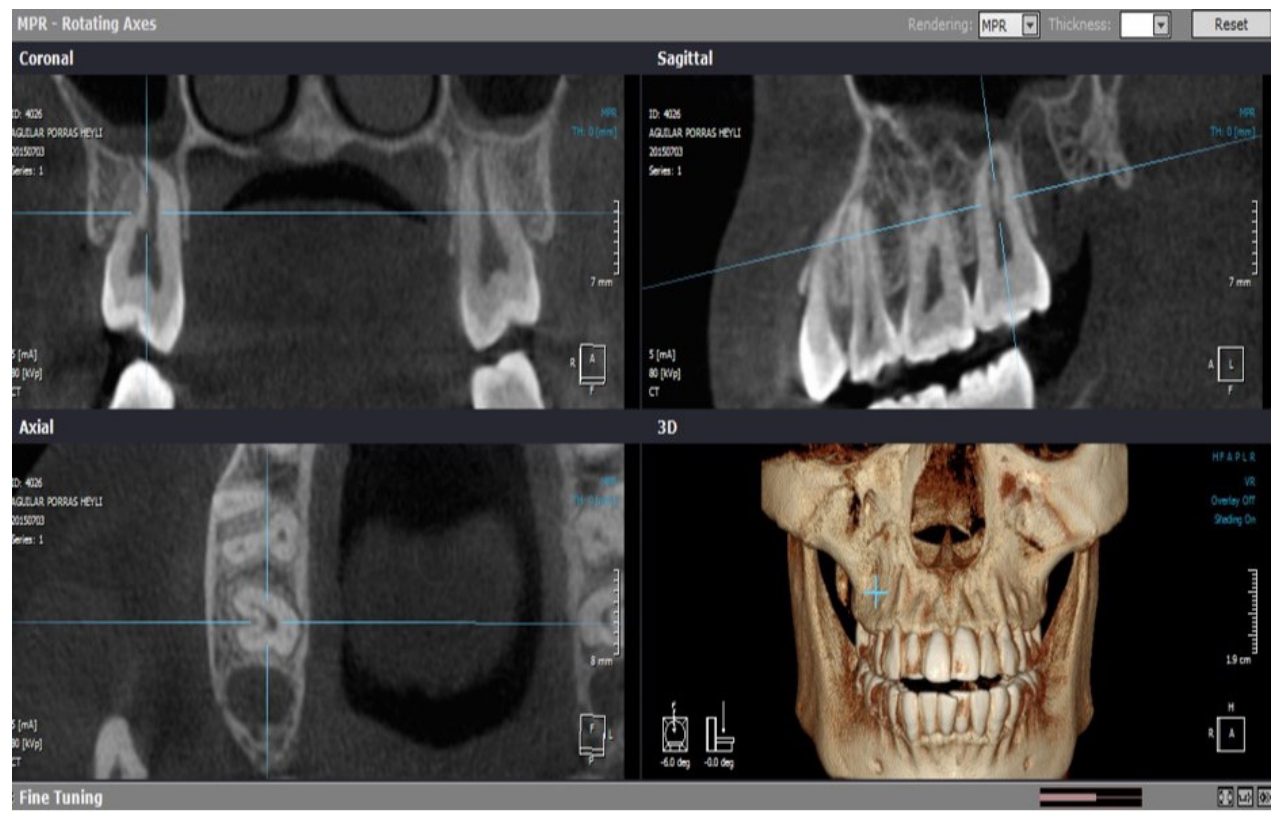

Figura 1. Variante 3:1 Una raíz 3 conductos, tercio cervical (pza. 17).

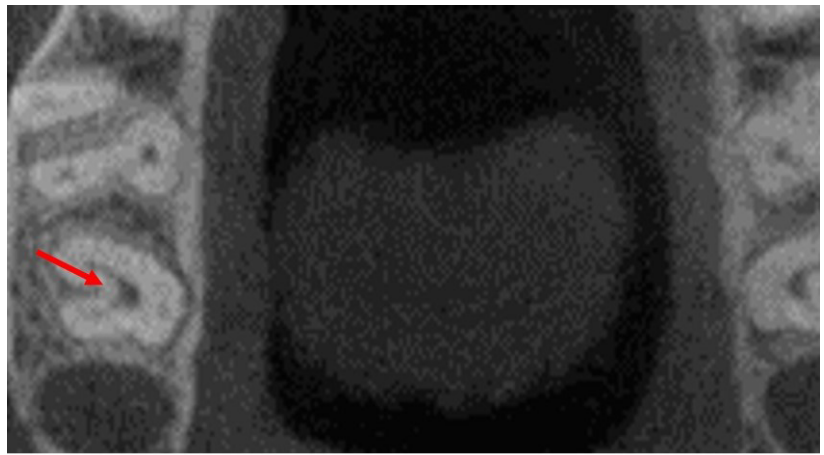

Figura 1.1 Corte axial, conducto en C (pza. 17).

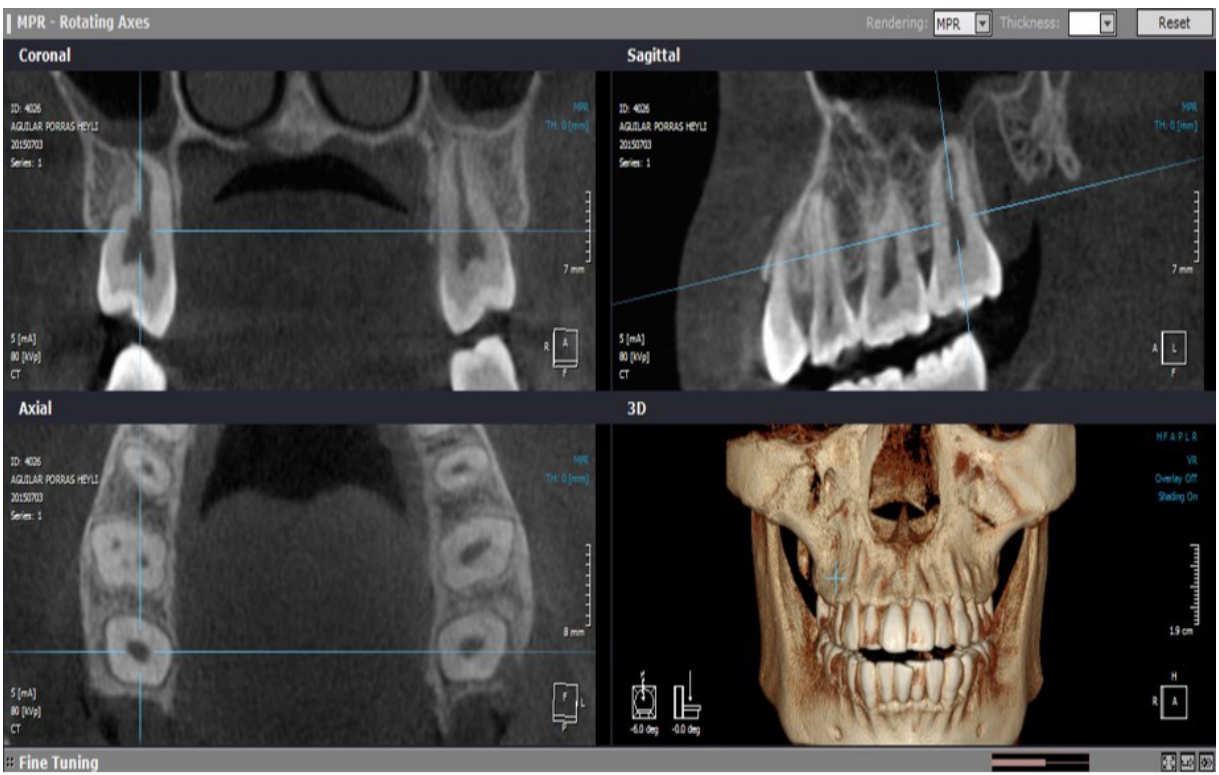

Figura 2. Variante 3:1 Una raíz 3 conductos, tercio medio (pza. 17). 


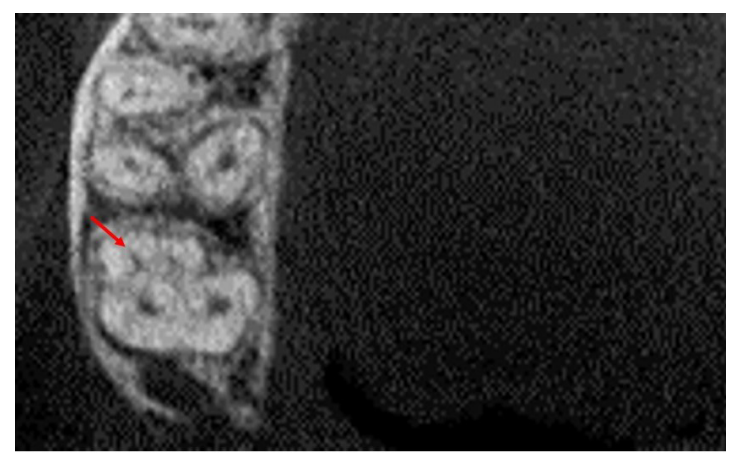

Figura 2.1 Corte axial, cuarto conducto (pza. 17).

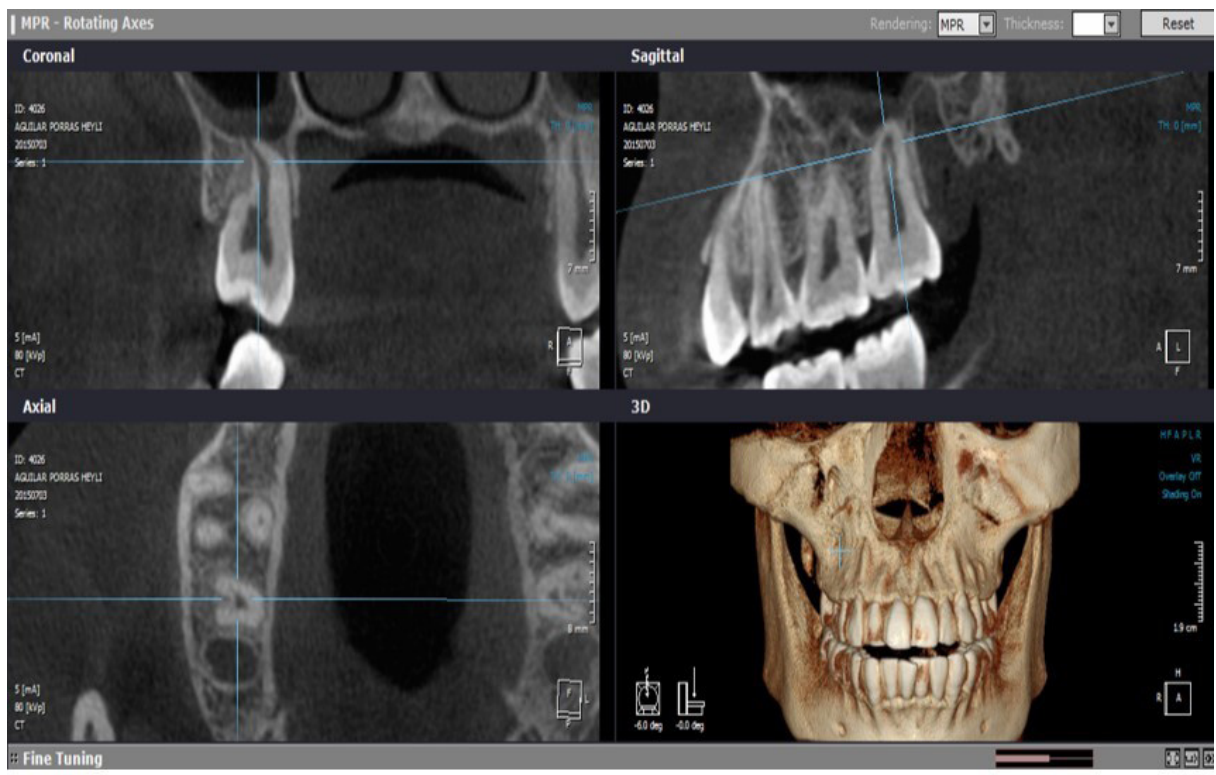

Figura 3. Variante 3:1 Una raíz 3 conductos, tercio apical (pza. 17).

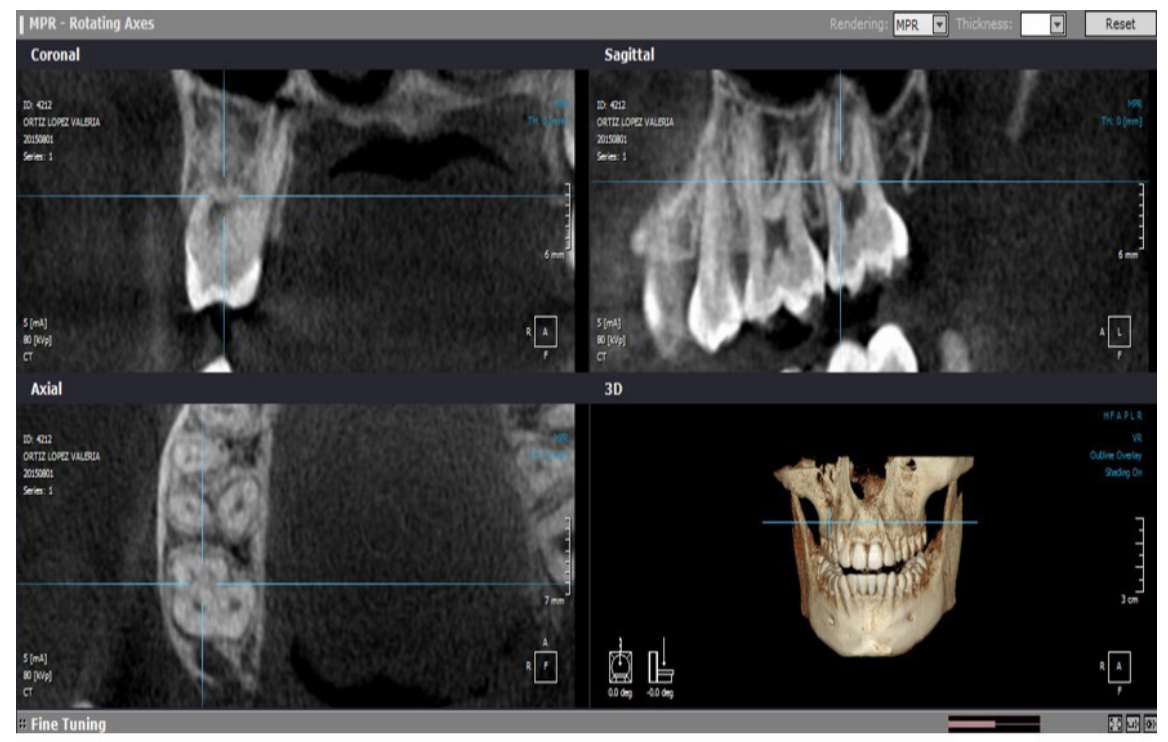

Figura 4. Variante 6:3 Raíces separadas: Vestibulomesial con 2 conductos vestibulodistal y palatino con un conducto en cada uno, tercio cervical (pza. 1.7). 
Para asegurar la confiabilidad y validez de la muestra obtenida, toda la información fue revisada por la investigadora y la gold standard especialista en radiología oral y maxilofacial. Cada raíz de cada pieza dental fue vista en los diferentes cortes axial, sagital y coronal, en los diferentes tercios: cervical, medio y apical.

- En el corte axial: número de conductos y raíces. (fig.1) Esta es la vista óptima para evaluar la presencia del cuarto conducto (fig. 2).

- En el corte sagital: Dirección de las raíces (fig. 3)

- En el corte coronal: trayectoria del cuarto conducto.

Se realizó el análisis mediante el programa estadístico SPSS y se realizó la prueba de Chi-cuadrado con la finalidad de identificar las asociaciones entre las variables de estudio.

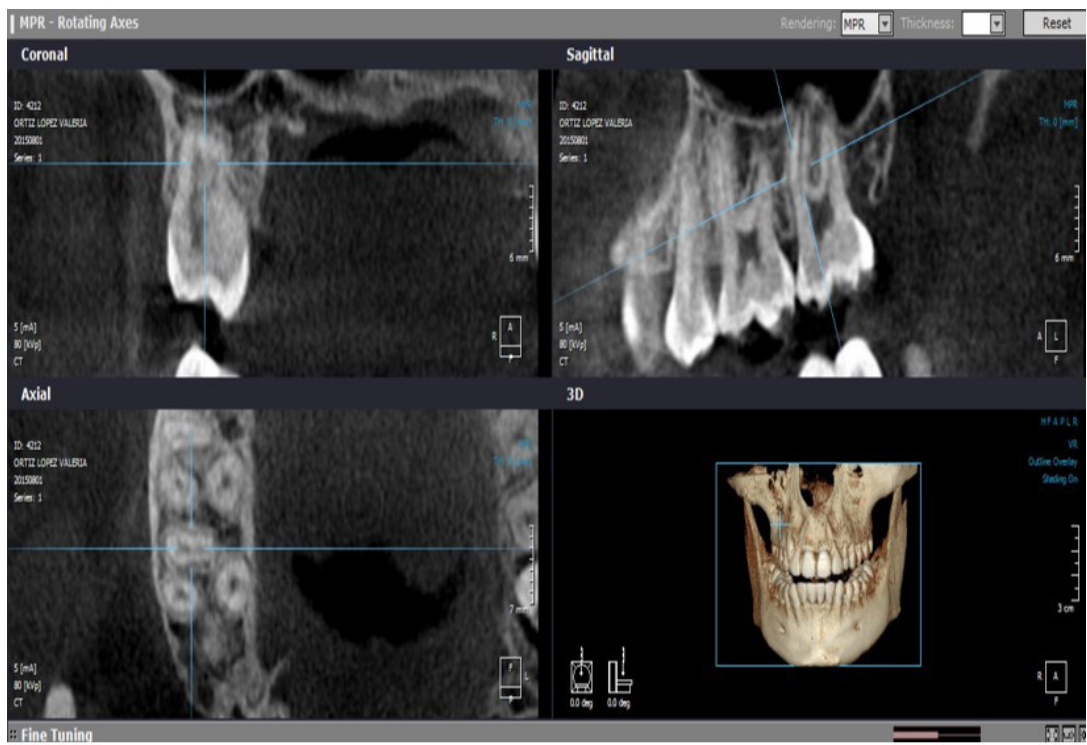

Figura 5. Variante 6:3 Raíces separadas: vestibulomesial con 2 conductos vestibulodistal y palatino con un conducto en cada uno, tercio medio (pza. 1.7).

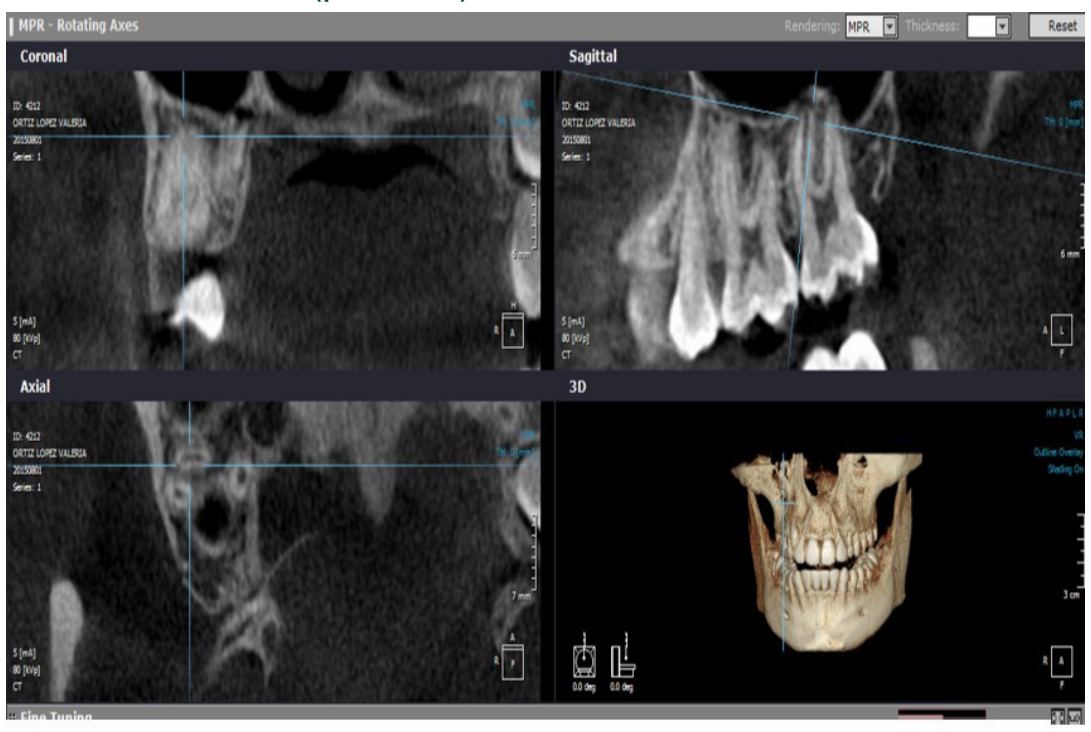

Figura 6. Variante 6:3 Raíces separadas: vestibulomesial con 2 conductos vestibulodistal y palatino con un conducto en cada uno, tercio apical (pza. 1.7). 


\section{RESULTADOS}

De las 170 segundas molares superiores y terceras molares superiores incluidas en el presente estudio, se observaron 7 variantes. Variante 1: una raíz con un conducto; Variante 2: una raíz con dos conductos; Variante 3: una raíz con tres conductos; Variante 4: 2 raíces separadas, una vestibular y una palatina, con un conducto en cada raíz; Variante 5: 3 raíces separadas MV, DV y palatino, con un conducto en cada uno; Variante 6: 3 raíces separadas, un conducto en palatino, un canal en DV y 2 conductos en MV; Variante 7:2 raíces separadas, vestibular (2 conductos) y palatino (1 conducto) y otros.

En las segundas molares superiores la variante 5 ( 3 raíces separadas mesiovestibular, distovestibular y palatino, con un conducto en cada uno) representa el 47,1\%; la variante 6 (3 raíces separadas, un conducto en palatino, un conducto el en distovestibular y 2 conductos en la raíz mesiovestibular) un 38,8\%. En las terceras molares superiores la variante 5 ( 3 raíces separadas mesiovestibular, distovestibular y palatino, con un conducto en cada uno) representa el $21,9 \%$; la variante 3 (una raíz con tres conductos) un $34,9 \%$. $p<0.001$ (Tabla 1).

El cuarto conducto en las segundas molares superiores se encontró en un $40 \%$ de los casos y en las terceras molares superiores estuvo presente en un $4,8 \%$. $\mathrm{p}<0.001$ (Tabla N²).

No se encontró asociación en bilateralidad ( $p=$ $0,931)$ respecto a número de conductos y raíces; en segundas molares superiores un $69,4 \%$ y en terceras molares superiores en $69,9 \%$. (Tabla 3). Respecto al sexo se encontró asociación estadísticamente significativa entre la presencia del cuarto conducto en segundas y terceras molares superiores. $p<0.001$ (Tabla 4).

\section{DISCUSIÓN}

El presente estudio proporciona una descripción anatómica detallada del segundo molar superior y de la tercera molar superior basada en un análisis retrospectivo de imágenes tomográficas de haz cónico volumétrico. La tomografía de haz cónico volumétrico es una herramienta esencial que proporciona al clínico información detallada que las radiografías intraorales convencionales o imágenes panorámicas no son capaces de mostrar, debido a su falta de capacidad para demostrar características anatómicas en tres dimensiones. Estos incluyen la determinación de la morfología, anomalías o conductos extras en una raíz. El éxito del tratamiento endodóntico exige limpieza, conformación y obturación del sistema de conductos radiculares por eso, los clínicos deben tener un conocimiento de la morfología del conducto radicular. No detectar y tratar el cuarto conducto resultará en una reducción en el pronóstico a largo plazo.

De acuerdo al número de raíces de segundas molares superiores a Kalender et al(13) encontraron entre 438 segundas molares superiores; 3,1\% tenían una sola raíz; 5,9\% tenían 2 raíces; 89,4\% tenían 3 raíces y $1,3 \%$ tenían 4 raíces. En el estudio de Singh \& Pawar encontraron18 de los 100 segundos molares superiores, $5 \%$ tenían una raíz y $95 \%$ tenían 3 raíces; Rohuani et al(19) encontraron 1,6\% molares superiores con 4 raíces. Felsypremila et al(20) hallaron $1,1 \%$ con 4 raíces; $8,9 \% 2$ raíces; 80,3\% 3 raíces; y un $9,7 \%$ una raíz. En el estudio de Mirzae et al(21) encontraron que todas las segundas molares tenían 3 raíces. Singh \& Pawar(18) hallaron $95 \%$ con 3 raíces y $5 \%$ una sola raíz. Un $85,07 \%$ presentó 3 raíces en un estudio de Nikoloudaki(15). Estrela et al(22) 69\% tenían 3 raíces, 29\% 2 raíces, y solo 2 dientes presentaron una sola raíz. 
Tabla 1. Frecuencia de la morfología interna en segundas y terceras molares superiores

\begin{tabular}{|c|c|c|c|c|c|c|}
\hline \multirow{2}{*}{$\begin{array}{c}\text { Tipo de } \\
\text { pieza }\end{array}$} & \multicolumn{2}{|c|}{$\begin{array}{c}\text { Variante 1: 1 raíz con 1 } \\
\text { conducto }\end{array}$} & \multicolumn{2}{|c|}{$\begin{array}{c}\text { Variante 1: } 1 \text { raíz con 2 con- } \\
\text { ductos }\end{array}$} & \multicolumn{2}{c|}{$\begin{array}{c}\text { Variante 1: } 1 \text { raíz con } 3 \text { con- } \\
\text { ductos }\end{array}$} \\
\cline { 2 - 7 } & $\mathrm{n}$ & $\%$ & $\mathrm{n}$ & $\%$ & $\mathrm{n}$ & $\%$ \\
\hline $\begin{array}{c}\text { Segunda } \\
\text { molar supe- } \\
\text { rior }\end{array}$ & 1 & 0,6 & 0 & 0,0 & 17 & 10,0 \\
\hline $\begin{array}{c}\text { Tercera mo- } \\
\text { lar superior }\end{array}$ & 41 & 28,1 & 6 & 4,1 & 51 & 34,9 \\
\hline Total & 42 & 13,3 & 6 & 1,9 & 68 & 21,5 \\
\hline
\end{tabular}

\begin{tabular}{|c|c|c|c|c|c|c|}
\hline \multicolumn{7}{|c|}{ Tabla 1 (Continuación) } \\
\hline $\begin{array}{c}\text { Tipo de } \\
\text { pieza }\end{array}$ & $\begin{array}{c}\text { Variante 4: 2 raíces separa- } \\
\text { das, 1 vestibular, 1 palatina } \\
\text { con 1 conducto en cada raíz }\end{array}$ & $\begin{array}{c}\text { Variante 5: 3 raíces separa- } \\
\text { das MV, DV y palatino con 1 } \\
\text { conducto en cada uno }\end{array}$ & $\begin{array}{c}\text { Variante 6: 3 raíces separa- } \\
\text { das, 1 conducto en palatino, } \\
\text { un conducto en DV y 2 } \\
\text { conductos en MV }\end{array}$ \\
\cline { 2 - 7 } & $\mathrm{n}$ & $\%$ & $\mathrm{n}$ & $\%$ & $\mathrm{n}$ & $\%$ \\
\hline $\begin{array}{c}\text { Segunda } \\
\text { molar supe- } \\
\text { rior }\end{array}$ & 1 & 0,6 & 80 & 47,1 & 66 & 38,8 \\
\hline $\begin{array}{c}\text { Tercera mo- } \\
\text { lar superior }\end{array}$ & 2 & 1,4 & 30 & 20,5 & 9 & 6,2 \\
\hline Total & 3 & 0,9 & 110 & 34,8 & 75 & 23,7 \\
\hline
\end{tabular}

\begin{tabular}{|c|c|c|c|c|c|c|}
\hline \multicolumn{7}{|c|}{ Tabla 1 (Continuación) } \\
\hline \multirow[t]{2}{*}{$\begin{array}{l}\text { Tipo de } \\
\text { pieza }\end{array}$} & \multicolumn{2}{|c|}{$\begin{array}{l}\text { Variante } 7: 2 \text { raíces separa- } \\
\text { das, vestibular( } 2 \text { conductos) } \\
\text { y palatino( } 1 \text { conducto) }\end{array}$} & \multicolumn{2}{|c|}{ Otros } & \multicolumn{2}{|c|}{ Total } \\
\hline & $n$ & $\%$ & $n$ & $\%$ & $\mathrm{n}$ & $\%$ \\
\hline $\begin{array}{l}\text { Segunda } \\
\text { molar supe- } \\
\text { rior }\end{array}$ & 3 & 1,8 & 2 & 1,2 & 170 & 100,0 \\
\hline $\begin{array}{l}\text { Tercera mo- } \\
\text { lar superior }\end{array}$ & 3 & 2,1 & 4 & 2,7 & 146 & 100,0 \\
\hline Total & 6 & 1,9 & 6 & 1,9 & 316 & 100,0 \\
\hline \multicolumn{7}{|l|}{$p=0,000$} \\
\hline Fuente: Elab & ción pr & & & & & \\
\hline
\end{tabular}




\begin{tabular}{|c|c|c|c|c|c|c|c|}
\hline \multicolumn{2}{|c|}{ Tabla 2. Frecuencia de cuarto conducto en segundas y terceras molares superiores } & \multicolumn{2}{c|}{ Presente } & \multicolumn{2}{c|}{ Total } & $\mathrm{p}$ \\
\cline { 2 - 7 } & $\mathrm{n}$ & $\%$ & $\mathrm{n}$ & $\%$ & $\mathrm{n}$ & $\%$ & \\
\hline $\begin{array}{c}\text { Segunda molar } \\
\text { superior }\end{array}$ & 102 & 60,0 & 68 & 40,0 & 170 & 100,0 & \multirow{2}{*}{0,000} \\
\hline $\begin{array}{c}\text { Tercera molar } \\
\text { superior }\end{array}$ & 137 & 93,8 & 9 & 6,2 & 146 & 100,0 & \\
\hline Total & 239 & 75,6 & 77 & 24,4 & 316 & 100,0 & \\
\hline
\end{tabular}

\begin{tabular}{|c|c|c|c|c|c|c|c|}
\hline \multicolumn{8}{|c|}{ Bilateralidad } \\
\hline & \multicolumn{2}{|c|}{ Sí } & \multicolumn{2}{|c|}{ No } & \multicolumn{2}{|c|}{ Total } & \multirow[t]{2}{*}{$\mathrm{p}$} \\
\hline & $\mathrm{n}$ & $\%$ & $\mathrm{n}$ & $\%$ & $\mathrm{n}$ & $\%$ & \\
\hline $\begin{array}{l}\text { Segunda molar } \\
\text { superior }\end{array}$ & 118 & 69,4 & 52 & 30,6 & 170 & 100,0 & \multirow{3}{*}{0,931} \\
\hline $\begin{array}{l}\text { Tercera molar } \\
\text { superior }\end{array}$ & 102 & 69,9 & 44 & 30,1 & 146 & 100,0 & \\
\hline Total & 220 & 69,6 & 96 & 30,4 & 316 & 100,0 & \\
\hline \multicolumn{7}{|c|}{ Fuente: Elaboración propia - Prueba Chi-cuadrado de Pearson } & \\
\hline
\end{tabular}

\section{Tabla 4. Frecuencia del cuarto conducto de acuerdo al sexo en segundas y terceras molares superiores}

\begin{tabular}{|c|c|c|c|c|c|c|c|c|}
\hline \multicolumn{9}{|c|}{ Presencia de MB2 } \\
\hline \multirow{2}{*}{ Género } & \multirow{2}{*}{ Tipo de pieza } & \multicolumn{2}{|c|}{ Ausente } & \multicolumn{2}{|c|}{ Presente } & \multicolumn{2}{|c|}{ Total } & \multirow[t]{2}{*}{$\mathrm{p}$} \\
\hline & & $\mathrm{n}$ & $\%$ & $\mathrm{n}$ & $\%$ & $\mathrm{n}$ & $\%$ & \\
\hline \multirow{2}{*}{ Femenino } & $\begin{array}{l}\text { Segunda molar } \\
\text { superior }\end{array}$ & 3 & 63,0 & 37 & 37,0 & 100 & 100 & \multirow{6}{*}{0,000} \\
\hline & $\begin{array}{l}\text { Tercera molar } \\
\text { superior }\end{array}$ & 96 & 98,0 & 2 & 2,0 & 98 & 100 & \\
\hline \multirow{2}{*}{ Masculino } & $\begin{array}{l}\text { Segunda molar } \\
\text { superior }\end{array}$ & 39 & 55,7 & 31 & 44,3 & 70 & 100 & \\
\hline & $\begin{array}{l}\text { Tercera molar } \\
\text { superior }\end{array}$ & 41 & 85,4 & 7 & 14,6 & 48 & 100 & \\
\hline \multirow{2}{*}{ Total } & $\begin{array}{c}\text { Segunda molar } \\
\text { superior }\end{array}$ & 102 & 60 & 68 & 40,0 & 170 & 100 & \\
\hline & $\begin{array}{l}\text { Tercera molar } \\
\text { superior }\end{array}$ & 137 & 93,8 & 9 & 6,2 & 146 & 100 & \\
\hline
\end{tabular}


En el presente estudio sobre las segundas molares 10,6\% tenían una sola raíz, 2,9\% tenían 2 raíces, $86,5 \%$ tenían 3 raíces y no hubo ninguna con 4 raíces. Respecto al número de raíces de terceras molares superiores según Singh \& Pawar $^{18}$ hallaron 20\% con una sola raíz, $33 \%$ con 2 raíces y $47 \%$ con 3 raíces, en el presente estudio se halló que de las terceras molares $69,2 \%$ tenían una sola raíz; 4,1\%, 2 raíces y $26,7 \%, 3$ raíces.

En segundas molares superiores en estudios con tomografía de haz cónico volumétrico como el de Zhang et al.(23) encontraron un 22\% de presencia del cuarto conducto, Abarca et al.(14) encontró un 42,48\%; Felsypremila(20), un 60,1\%; Betancourt(24), un $48 \%$ y Domark et al.(25), 57\%. En estudios de diafanización Singh \& Pawar(18) encontraron presencia del cuarto conducto un $18 \%$, en un estudio de Imura et al.(26) encontraron un 40\%. El presente estudio muestra un $40 \%$ de existencia del cuarto conducto.

Respecto a la presencia del cuarto conducto en terceras molares superiores Pécora et al.(1) encontró un $32 \%$ y en el estudio de Singh \& Pawar(18) un $40.4 \%$ ambas investigaciones usaron como método la diafanización. En el presente estudio se halló un 4.8\% de presencia de cuarto conducto en la raíz mesiovestibular de terceras molares superiores. Mayores porcentajes reportados por otros estudios podrían explicarse con el método en este caso la diafanización que es el gold standard y además estudios ex vivo sobre la incidencia de conductos adicionales revelan mayor detección que en estudios in vivo. Estas diferencias de frecuencia revelan la variabilidad de la raíz mesial en los molares maxilares, podría explicarse por el tamaño de la muestra, la observación y la etnia de los pacientes.

Felsypremila et al.(20) mostraron que la bilateralidad de acuerdo al número de conductos y raíces fue
$70,8 \%$ en segundas molares superiores, Zhang et al.(23) reportaron un 84\%. Asimismo, Betancourt(24) no encontró diferencias en la hemiarcada. En el presente estudio no se encontró bilateralidad $p=0.931$.

Kalender et al.(13) encontró un 0,22\% en conducto en $C$ entre 438 segundos molares maxilares en una población turco chipriota. Felsypremila et al.(20) encontró $5,6 \%$ de conducto en $C$ en segundas molares superiores. Singh \& Pawar(18) no encontraron conductos en $C$ en segundas ni terceras molares superiores. Silva et al.(16) no encontró conductos en C en segunda molares. En el presente estudio se encontró $7,6 \%$ en segundas molares superiores y en terceras molares superiores un 20,5\% de conductos en forma de C. Esta variación puede ser causada por diferencias en el tamaño de la muestra, métodos, y / o la diversidad de la población.

También se encontraron otros tipos de morfología se encontraron 2 piezas en segundas molares superiores con 2 raíces y 3 conductos, 1 vestibular y el conducto palatino y el conducto distal se unen, también se encontró una tercera molar con un conducto en $C$ y una raíz extra vestibular y otra tercera molar superior mostró una raíz con 4 conductos, dos mesiales y dos distales. También se encontró la presencia de 3 conductos en la raíz mesiovestibular en 2 segundas molares superiores, de un mismo paciente, demostrando bilateralidad.

Varios estudios demostraron la calidad de la imagen de tomografía de haz cónico volumétrico y su aplicación para estudiar la morfología del conducto radicular antes del tratamiento clínico de la endodoncia. Sin embargo, el uso de la tomografía de haz cónico volumétrico debe ser indicada solamente en los casos en que la anatomía y/o morfología sea compleja. Vale la pena recordar que la imagen de tomografía de haz cónico volumétrico todavía utiliza radiación 
ionizante. Aunque mínimas, siempre debe tener en cuenta el protocolo. Los casos de endodoncia se deben juzgar individualmente, y los pacientes siempre deben estar expuestos a la menor cantidad de radiación posible. En los casos en que una anatomía compleja inesperada se visualice después del acceso o cuando no se encuentran conductos, la imagen tomografía de haz cónico volumétrico intraoperatoria es siempre una excelente posibilidad.

\section{CONCLUSIONES}

1.- La frecuencia de la morfología interna en segundas molares superiores más prevalente fue de tres raíces separadas con un conducto en cada uno $47,1 \%$. En la tercera molar superior; una raíz con 3 conductos $34,9 \%$.

2.- La frecuencia de cuarto conducto en segundas molares superiores fue de $40 \%$ y en terceras molares superiores $4,8 \%$.
Aunque la tecnología de imágenes de tomografía de haz cónico volumétrico desempeña un papel importante en el campo de la endodoncia no se puede utilizar de forma rutinaria en la práctica clínica diaria. El clínico debe tener en cuenta que el paciente todavía está expuesto a la radiación ionizante y debería evaluar el riesgo relativo. Es de vital importancia para el paciente la exposición a la radiación ser tan bajo como sea razonablemente posible.

3.- La frecuencia de la bilateralidad en segundas molares superiores fue de $69,4 \%$ y en terceras molares superiores $69,9 \%$.

4.- La frecuencia de cuarto conducto de acuerdo al sexo en segundas molares superiores fue de $37 \%$ (femenino) y 44,3\% (masculino). En terceras molares superiores; $2,0 \%$ (femenino) y $10,4 \%$ (masculino).

\section{REFERENCIAS BIBLIOGRÁFICAS}

1. Pécora J, Woelfel J, Soussa Neto M, Issa E. Morphological study of the maxillary molars part II: internal anatomy. Braz Dent J. 1992; 3: 53-57.

2. Cantarore G, Berutti E, Castellucci A. Missed anatomy: frequency and clinical impact. Endod Topics. 2009; $15:$ 3-31.

3. Yoshioka T, Kikuchi I, Fukumoto Y, Kobayashi S, Suda H. Detection of the second mesiobuccal canal in mesiobuccal roots of maxillary molar teeth ex vivo. J Endod. 2005; 38: 124-128.

4. $\mathrm{Ng} \mathrm{Y,} \mathrm{Aung} \mathrm{H,} \mathrm{Alavi} \mathrm{A,} \mathrm{Gulabivala} \mathrm{K.} \mathrm{Root} \mathrm{and} \mathrm{canal} \mathrm{morphology} \mathrm{of} \mathrm{Burmese} \mathrm{maxillary} \mathrm{molars.} \mathrm{J} \mathrm{Endod.} \mathrm{2001;} \mathrm{34:} \mathrm{620-630.}$

5. Al Shalabi R, Omer O, Glennon J, Jennings M, Claffey N. Root canal anatomy of maxillary first and second permanent molars. J Endod. 2000; 33: 405-14.

6. Imura N, Hata G, Toda T, Otani S, Fagundes M. Two canals in mesiobuccal roots of maxillary molars. Int Endod J. 1998; $31: 410$.

7. Karaman G, Onay E, Ungor M, Colak M. Evaluating the potential key factors in assessing the morphology of mesiobuccal canal in maxillary first and second molars. Aust Endod J. 2011; 37: 134-140.

8. Tuncer A, Haznedaroglu F, Sert S. The location and accessibility of the second mesiobuccal canal in maxillary first molar. Eur J Dent. 2010; 4: 12-6. 
9. Domark J, Hatton J, Benison R. An ex vivo comparison of digital radiography, cone Beam and micro computed tomography in the detection of the number of canals in the mesiobuccal roots of maxillary molars. J Endod. 2013; 39 (7): $901-5$.

10.Cotton T, Geisler T, Holden D, Schwartz S, Schindler W. Endodontic applications of cone-beam volumetric tomography. J Endod. 2007; 33 (9): 1121-32.

11. Matherne R, Angelopoulos C, Kulild J, Tira D. Use of cone-beam computed tomography to identify root canal systems in vitro. J Endod. 2008; 34 (1): 87-9.

12.Peters $\mathrm{C}$, Peters $\mathrm{O}$. Cone beam computed tomography and other imaging techniques in the determination of periapical healing. Endod Topics. 2012; 26: 57-75.

13.Kalender A, Celikten B, Tufenkci P, Aksoy U, Basmaci F, Kelahmet U, Orhan K. Cone beam computed tomography evaluation of maxillary molar root canal morphology in a Turkish Cypriot population. Biotechnol. Biotechnol. Equip. $2015 ; 30$ (1): $145-50$.

14.Abarca J, Gómez B, Zaror C, Monardes H, Bustos L, Cantin M. Assessment of mesial root morphology and frequency of mb2 canals in maxillary molars using cone beam computed tomography. Int J Morphol.2015; 33 (4): 1333-7.

15.Nikoloudaki G, Kontogiannis T, Kerezoudis N. Evaluation of the root and canal morphology of maxillary permanent molars and the incidence of the second mesiobuccal root canal in Greek population using cone-beam computed tomography. Open Dent J. 2015; 9: 267-72.

16. Silva E, Nejaim Y, Haiter-Neto F, Cohenca N. Evaluation of root canal configuration of maxillary molars in a Brazilian population using cone-beam computed tomographic imaging: An in vivo study. J Endod. 2014; 40 (2): 173-6.

17. Altunsoy M, Ok E, Nur B, Aglarci O, Gungor E, Colak M. Root canal morphology analysis of maxillary permanent first and second molars in a southeastern Turkish population using cone-beam computed tomography. JDS. 2014; 10(4): 1-7.

18.Singh S, Pawar M. Root canal morphology of South Asian Indian maxillary molar teeth. Eur J Dent. 2015; 9: 133-44.

19. Rouhani A, Bagherpour A, Akbari M, Azizi M, Nejat A, Naghavi N. Cone-beam computed tomography evaluation of maxillary first and second molars in Iranian population: A morphological study. Iran Endod J. 2014; 9 (3): 190-4.

20. Felsypremila G, Vinothkumar TS, Kandaswamy D. Anatomic symmetry of root and root canal morphology of posterior teeth in Indian subpopulation using cone beam computed tomography: A retrospective study. Eur J Dent. 2015; 9: 500-7.

21.Mirzaie M, Tork Zaban P, Mohammadi V. Cone-beam computed tomography study in a Hamadani population in Iran.

22.Estrela C, Bueno M, Couto G, Rabelo L, Alencar A, Silva R, Pécora J et al. Study of root canal anatomy in human permanent Teeth in a subpopulation of Brazil's center region using cone-beam computed tomography - part 1. Braz Dent J. 2015; 26(5): 530-536

23.Zhang R, Yang H, Yu X, Wang H, Hu T, Dummer P. Use of CBCT to identify the morphology of maxillary permanent molar teeth in a Chinese subpopulation. J Endod. 2011;44: 162-9.

24.Betancourt P, Navarro P, Cantín M, Fuentes R. Cone-beam computed tomography study of prevalence and location of MB2 canal in the mesiobuccal root of the maxillary second molar. Int J Clin Exp Med. 2015; 8 (6): 9128-34.

25.Domark J, Hatton J, Benison R. An ex vivo comparison of digital radiography, cone Beam and micro computed tomography in the detection of the number of canals in the mesiobuccal roots of maxillary molars. J Endod. 2013; 39 (7): $901-5$.

26.Imura N, Hata G, Toda T, Otani S, Fagundes M. Two canals in mesiobuccal roots of maxillary molars. Int Endod J. $1998 ; 31: 410-4$.

Recibido: 20 de agosto de 2017

Aceptado: 28 de noviembre de 2017

Correspondencia: gisella@hotmail.com 